Commun.Fac.Sci.Univ.Ank.Series A 1

Volume 56, Number 1, Pages 1-6 (2007)

ISSN $1303-5991$

\title{
THE DISCRIMINANT OF SECOND FUNDAMENTAL FORM
}

\author{
BENGU KILIC
}

\begin{abstract}
In this study we consider the discriminant of the second fundamental form. As application we also give necessary condition for Vranceanu surface in $\mathbb{E}^{4}$ to have vanishing discriminant.
\end{abstract}

\section{Introduction}

Let $M$ be an $n$-dimensional Riemannian manifolds. For the vector fields $X, Y, Z$ on $M$ the curvature tensor $R$ of $M$ is defined by

$$
R(X, Y) Z=\nabla_{X}\left(\nabla_{Y} Z\right)-\nabla_{Y}\left(\nabla_{X} Z\right)-\nabla_{[X, Y]} Z
$$

where $\nabla$ is the Levi-Civita connection of $M$, and $[$,$] is Lie parantheses operator.$

Given a point $p \in M$ and a two-dimensional subspace $\sigma \subset T_{p} M$, the real number

$$
K(\sigma)=\frac{g(R(X, Y) X, Y)}{g(X, X) g(Y, Y)-g(X, Y)^{2}}
$$

is called the Sectional Curvature of $\sigma$ at point p, where $X, Y$ is any basis of $\sigma$ [1].

Let $f: M \rightarrow \widetilde{M}$ be an isometric immersion of an $n$-dimensional connected Riemannian manifold $M$ into an $m$-dimensional Riemannian manifold $\widetilde{M}$. For all local formulas and computations, we may assume $f$ as an imbedding and thus we shall often identify $p \in M$ with $f(p) \in \widetilde{M}$. The tangent space $T_{p} M$ is identified with a subspace $f_{*}\left(T_{p} M\right)$ of $T_{p} \widetilde{M}$ where $f_{*}$ is the differential map of $f$. Letters $X$, $Y$ and $Z$ (resp. $\zeta, \mu$ and $\xi$ ) vector fields tangent (resp. normal) to $M$. We denote the tangent bundle of $M$ (resp. $\widetilde{M}$ ) by $T M$ (resp. $T \widetilde{M}$ ), unit tangent bundle of $M$ by $U M$ and the normal bundle by $T^{\perp} M$. Let $\tilde{\nabla}$ and $\nabla$ be the Levi-Civita connections of $\widetilde{M}$ and $M$, resp. Then the Gauss formula is given by

$$
\tilde{\nabla}_{X} Y=\nabla_{X} Y+h(X, Y)
$$

Received by the editors March 21, 2006; Rev.: Feb. 23, 2007; Accepted: Feb. 26, 2007.

2000 Mathematics Subject Classification. 53C40,

Key words and phrases. Second fundamental form, discriminant. 
where $h$ denotes the second fundamental form. If the Weingarten formula is given by

$$
\tilde{\nabla}_{X} \xi=-A_{\xi} X+D_{X} \xi
$$

where $A$ denotes the shape operator and $D$ the normal connection. Clearly $h(X, Y)=$ $h(Y, X)$ and $A$ is related to $h$ as $\left\langle A_{\xi} X, Y\right\rangle=\langle h(X, Y), \xi\rangle$, where $\langle$,$\rangle denotes the$ Riemannian metrics of $M$ and $\widetilde{M}$.

For the second fundamental form, we define their covariant derivatives by

$$
\left(\bar{\nabla}_{X} h\right)(Y, Z)=D_{X}(h(Y, Z))-h\left(\nabla_{X} Y, Z\right)-h\left(Y, \nabla_{X} Z\right)
$$

where $X, Y, Z$ tangent vector fields over $M$ and $\bar{\nabla}$ is the van der Waerden Bortolotti connection [1].The equation of Codazzi implie, that $\bar{\nabla} h$ is symmetric hence

$$
\left(\bar{\nabla}_{X} h\right)(Y, Z)=\left(\bar{\nabla}_{Y} h\right)(X, Z)=\left(\bar{\nabla}_{Z} h\right)(X, Y)
$$

If $\bar{\nabla} h=0$ then the second fundamental form of $M$ is called parallel [7] (i.e. $M$ is 1-parallel) [4].

\section{Discriminant of The Second Fundamental Form}

Let $f: M \rightarrow \widetilde{M}$ be an isometric immersion of an $n$-dimensional connected Riemannian manifold $M$ into an $m$-dimensional Riemannian manifold $\widetilde{M}$. The main invariant of the second fundamental form $h$ is its discriminant $\Delta$, (see [2]) the real valued function on the planes (through 0 ) in $T_{x} M$ such that if the linearly independent tangent vectors $X, Y$ span $\sigma$, then

$$
\Delta_{X Y}=\Delta(\sigma)=\frac{\langle h(X, X), h(Y, Y)\rangle-\|h(X, Y)\|^{2}}{\|X\|^{2}\|Y\|^{2}-\langle X, Y\rangle^{2}} .
$$

For an isometric immersion $f: M \rightarrow \widetilde{M}$, the Gauss equation asserts that

$$
K(\sigma)=\Delta(\sigma)+\widetilde{K}(d f(\sigma))
$$

where $K$ and $\widetilde{K}$ are the sectional curvatures of $M$ and $\widetilde{M}$, and $\sigma$ is any plane tangent to $M[6]$.

If the vectors in $T_{x} M$ are orthonormal then, the formula (2.1) reduces to

$$
\Delta_{X Y}=\langle h(X, X), h(Y, Y)\rangle-\|h(X, Y)\|^{2}
$$

Definition 2.1. We say that $h$ is $\lambda$-isotropic provided that $\|h(X, X)\|=\lambda$ for all unit vectors $X$ in $T_{x} M$. Clearly, an isometric immersion is isotropic provided that all its normal curvature vectors have the same length [5].

Lemma 2.2. [5] Suppose that $h$ is $\lambda$-isotropic on $T_{x} M$ and let $X, Y$ be orthonormal vectors in $T_{x} M$. Then

$$
\langle h(X, X), h(Y, Y)\rangle+2\|h(X, Y)\|^{2}=\lambda^{2} .
$$


The assertation (2.1) in the preceding lemma yields the following result.

Lemma 2.3. [5] If $h$ is $\lambda$ - isotropic then for orthonormal vectors $X, Y$ in $T_{x} M$

i) $\Delta_{X Y}+3\|h(X, Y)\|^{2}=\lambda^{2}$.

ii) $2 \Delta_{X Y}+\lambda^{2}=3\langle h(X, X), h(Y, Y)\rangle$.

In the case of $\widetilde{M}=\mathbb{E}^{n+d}$ the sectional curvature $K(\sigma)$ of $M$ reduces to

$$
K(\sigma)=\Delta_{X Y}
$$

Remark 2.4. Let $K$ be a Gaussian curvature of the surface $M \subseteq \mathbb{E}^{m}$. Then $K=$ $\Delta_{X Y}$. If $\Delta_{X Y}=0$ then $M$ is said to be flat.

Proposition 1. [7] Let $f: M^{2} \rightarrow \mathbb{E}^{2+d}$ be isometric immersion. If the second fundamental form of $M^{2}$ is 1-parallel (i.e. $\bar{\nabla} h=0$ ) then $f\left(M^{2}\right)$ is one of the following surfaces

i) $\mathbb{E}^{2}$

ii) $S^{2} \subset \mathbb{E}^{3}$

iii) $I R^{1} \times S^{1} \subset \mathbb{E}^{3}$

iv) $S^{1}(a) \times S^{1}(b) \subset \mathbb{E}^{4}$

v) $V^{2} \subset \mathbb{E}^{5}$.

Proposition 2. Let $M$ be a ruled surface of the form

$$
x(u, v)=\beta(u)+v \delta(u) .
$$

If $\Delta x y=0$ (i.e $M$ is flat) then $M$ is one of the following surfaces;

i) a cone of the form $x(u, v)=p+v \delta(u)$ or,

ii) a cylinder of the form $x(u, v)=\beta(u)+v q$ or,

iii) a tangent developable surface of the form $x(u, v)=\beta(u)+v \beta^{\prime}(u),(v>0)$.

Proof. (see [6]).

For more details for the following Examples see [3].

Example 2.5. For the following surfaces $K=\Delta_{X Y}=0$;

1) The torus $T^{2}$ embedded in $\mathbb{E}^{4}$ by

$$
T^{2}=\{(\cos \theta, \sin \theta, \cos \varphi, \sin \varphi): \theta, \varphi \in I R\}
$$

2) The helicel cylinder $H^{2}$ embedded in $\mathbb{E}^{4}$ by

$$
H^{2}=\{(u, c \cos v, c \sin v, d v): u, v \in I R\}
$$

3)The cylinder $C$ embedded in $\mathbb{E}^{3}$ by

$$
C=\{(a \cos s, a \sin s, t): s, t \in I R\} .
$$


Example 2.6. For the following surfaces $K=\Delta_{X Y} \neq 0$;

1) The sphere $S^{2}$ embedded in $\mathbb{E}^{3}$ by

$$
\begin{aligned}
S^{2} & =\{(a \cos s \cos t, a \cos s \sin t, a \sin s): s, t \in I R\}, \\
\Delta_{X Y} & =\frac{1}{a^{2}} .
\end{aligned}
$$

2)The helicoid $H$ embedded in $\mathbb{E}^{3}$ by

$$
\begin{aligned}
H & =\{(s \cos t, s \sin t, a t): s, t \in I R\} \\
\Delta_{X Y} & =-\frac{a^{2}}{\left(s^{2}+a^{2}\right)^{2}} .
\end{aligned}
$$

Proposition 3. The Veronese surface parametrized by

$$
V^{2}=\left\{\frac{1}{\sqrt{3}} y z, \frac{1}{\sqrt{3}} z x, \frac{1}{\sqrt{3}} x y, \frac{1}{2 \sqrt{3}}\left(x^{2}-y^{2}\right), \frac{1}{6}\left(x^{2}+y^{2}-2 z^{2}\right)\right\}
$$

is spherical.

Proof. The parametric representation of $V^{2}$ defines an isometric immersion of $S^{2}(\sqrt{3})$ into $S^{4}(1)$. Two points $(x, y, z)$ and $(-x,-y,-z)$ of $S^{2}(\sqrt{3})$ are mapped into the same point of $S^{4}(1)$, and this mapping defines an imbedding of the real projective plane into $S^{4}(1)$. This real projective plane imbedded in $S^{4}(1)$ is called the Veronese surface [1] which is minimal in $S^{4}(1) \subset \mathbb{E}^{5}$.

A submanifolds (or immersion) is called non-spherical in the fact that it does not lie in a sphere.

Theorem 2.7. Let $f: M^{n} \rightarrow \mathbb{E}^{n+d}$ be non-spherical isometric immersion. If $M$ is 1-parallel then $\Delta_{X Y}=0$.

Proof. Since $f(M)$ is not spherical therefore by Proposition 3 the possible nonspherical 1-parallel surfaces are cylinder $I R^{1} \times S^{1} \subset \mathbb{E}^{3}$ and torus $S^{1}(a) \times S^{1}(b) \subset \mathbb{E}^{4}$. On the other hand, both of them have vanishing sectional curvature.

Definition 2.8. The Vranceanu surface is defined by the parametrized

$$
x(s, t)=\{u(s) \cos s \cos t, u(s) \cos s \sin t, u(s) \sin s \cos t, u(s) \sin s \sin t\} .
$$

Theorem 2.9. Let the Vranceanu surface is given by the parametrized (2.6). The Vranceanu surface has vanishing Gaussian curvature $\left(K=\Delta_{X Y}=0\right)$ if and only if $\left(u^{\prime}\right)^{2}-u u^{\prime \prime}=0$ (i.e. $u=C e^{k s}$ for the real constant $0 \neq C$ and $k$ ). 
Proof. We choose a moving frame $e_{1}, e_{2}, e_{3}, e_{4}$ such that $e_{1}, e_{2}$ are tangent to $M$ and $e_{3}, e_{4}$ are normal to $M$ as given by the following

$$
\begin{aligned}
e_{1} & =(-\cos s \sin t, \cos s \cos t,-\sin s \sin t, \sin s \cos t) \\
e_{2} & =\frac{1}{A}(B \cos t, B \sin t, C \cos t, C \sin t) \\
e_{3} & =\frac{1}{A}(-C \cos t,-C \sin t, B \cos t, B \sin t) \\
e_{4} & =(-\sin s \sin t, \sin s \cos t, \cos s \sin t,-\cos s \cos t)
\end{aligned}
$$

where we put $A=\sqrt{u^{2}+\left(u^{\prime}\right)^{2}}, B=u^{\prime} \cos s-u \sin s, C=u^{\prime} \sin s+u \cos s$. Then we have

$$
e_{1}=\frac{1}{u} \frac{\partial}{\partial t}, e_{2}=\frac{1}{A} \frac{\partial}{\partial s} .
$$

Then the structure equations of $\mathbb{E}^{m}$ are obtained as follows:

$$
\begin{aligned}
\tilde{\nabla}_{e_{i}} e_{j} & =w_{j}^{k}\left(e_{i}\right) e_{k}+h_{i j}^{\alpha} e_{\alpha}, 1 \leq i, j, k \leq 2 \\
\tilde{\nabla}_{e_{i}} e_{\alpha} & =-h_{i j}^{\alpha} e_{j}+w_{\alpha}^{\beta}\left(e_{i}\right) e_{\beta}, 3 \leq \alpha, \beta \leq 4 \\
D_{e_{\alpha}} e_{\beta} & =w_{\alpha}^{\beta}\left(e_{i}\right) e_{\beta}
\end{aligned}
$$

where $D$ is the normal connection and $h_{i j}^{\alpha}$ the coefficients of the second fundamental form $h$. Using (2.7), (2.8), (2.9) and (2.10) we can get that the coefficients of the second fundamental form $h$ and the connection form $w_{B}^{A}$ are as following:

$$
\begin{aligned}
& h_{11}^{3}=\frac{1}{\sqrt{u^{2}+\left(u^{\prime}\right)^{2}}}=\alpha, h_{12}^{3}=h_{21}^{3}=0 \\
& h_{22}^{3}=\frac{2\left(u^{\prime}\right)^{2}-u u^{\prime \prime}+u^{2}}{\left(u^{2}+\left(u^{\prime}\right)^{2}\right)^{3 / 2}}=\beta \\
& h_{11}^{4}=h_{22}^{4}=0, h_{12}^{4}=h_{21}^{4}=-\frac{1}{\sqrt{u^{2}+\left(u^{\prime}\right)^{2}}} .
\end{aligned}
$$

The Gauss curvature is given by

$$
\begin{aligned}
K & =\operatorname{det}\left(h_{i j}^{3}\right)+\operatorname{det}\left(h_{i j}^{4}\right), 1 \leq i, j \leq 2 \\
& =\frac{\left(u^{\prime}\right)^{2}-u u^{\prime \prime}}{\left(u^{2}+\left(u^{\prime}\right)^{2}\right)^{2}} .
\end{aligned}
$$

Thus this completes the proof of the theorem. 
ÖZET: Bu çalışmada, ikinci temel formun diskriminantı gözönünde bulunduruldu. $\mathbb{E}^{4}$ de Vranceanu yüzeyinin sıfır diskriminantlı olması için gerekli koşul verildi.

\section{REFERENCES}

[1] B.Y.Chen, Geometry of Submanifolds, (1973).

[2] S.Fueki, Helices and Isometric Immersions, Tsukuba J.Math.Vol 22, No 2 (1998),427-445.

[3] B.Kilic, Sonlu Tipte Egriler ve Yüzeyler, PhD Thesis, Hacettepe Üniversitesi, (2002).

[4] U. Lumiste, Submanifolds with parallel fundamental form, Handbook of Differential Geometry , (1999), Vol 1, Chapter 7, 86 p.

[5] B. O,Neill, Isotropic and Kahler Immersions, Canadian J. Math. 17(1965), 907-915.

[6] B. O,Neill, Elementary Differential Geometry,(1966).

[7] R.Walden, Untermannigfaltigkeiten mit Paralleler Zweiter Fundamentalform in Euklidischen Räumen und Sphären, Manuscripta Math.10,91-102 (1973).

Current address: Balikesir University,Faculty of Art and Sciences, Department of Mathematics, Balikesir, TURKEY.

E-mail address: benguk@balikesir.edu.tr

$U R L$ : http://math.science.ankara.edu.tr 